\title{
Competing Sellers in Online Markets: Reserve Prices, Shill Bidding, and Auction Fees
}

\author{
Enrico H. Gerding, Alex Rogers, Rajdeep K. Dash and Nicholas R. Jennings \\ University of Southampton, Southampton, SO17 1BJ, UK. \\ $\{$ eg,acr,rkd02r,nrj\}@ecs.soton.ac.uk
}

\begin{abstract}
In this paper, we consider competition between sellers offering similar items in concurrent online auctions, where each seller must set its individual auction parameters (such as the reserve price) in such a way as to attract buyers. We show that there exists a pure Nash equilibrium in the case of two sellers with asymmetric production costs. In addition, we show that, rather than setting a reserve price, a seller can further improve its utility by shill bidding (i.e., pretending to be a buyer in order to bid in its own auction). But, using an evolutionary simulation, we show that this shill bidding introduces inefficiences within the market. However, we then go on to show that these inefficiences can be reduced when the mediating auction institution uses appropriate auction fees that deter sellers from submitting shill bids.
\end{abstract}

\section{Categories and Subject Descriptors}

I.2.11 [Distributed Artificial Intelligence]

\section{General Terms}

Economics

\section{Keywords}

Mechanism Design, Competition, Shill Bidding, Auction Fees

\section{INTRODUCTION}

Online markets are becoming increasingly prevalent and extend to a wide variety of areas such as e-commerce, Grid computing, recommender systems, and sensor networks. To date, much of the existing research has focused on the design and operation of individual auctions or exchanges for allocating goods and services. In practice, however, similar items are typically offered by multiple independent sellers that compete for buyers and set their own terms and conditions (such as their reserve price and the type and duration of the auction) within an institution that mediates between buyers and sellers. Examples of such institutions include eBay, Amazon and Yahoo!, where at any point in time multiple concurrent auctions with different settings are selling

Permission to make digital or hard copies of all or part of this work for personal or classroom use is granted without fee provided that copies are not made or distributed for profit or commercial advantage and that copies bear this notice and the full citation on the first page. To copy otherwise, to republish, to post on servers or to redistribute to lists, requires prior specific permission and/or a fee.

AAMAS'06 May 8-12 2006, Hakodate, Hokkaido, Japan.

Copyright 2006 ACM 1-59593-303-4/06/0005 ...\$5.00.

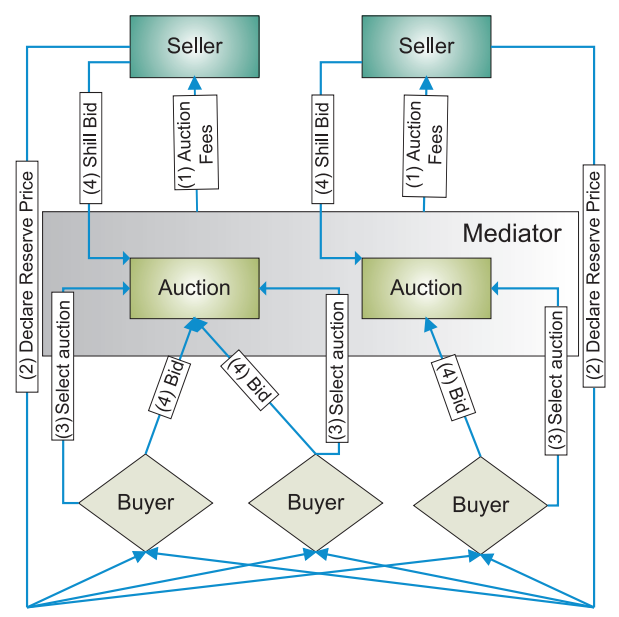

Figure 1: The competing sellers game.

similar objects, often resulting in strong competition. Given this competition, a key research question is how a seller should select their auction settings in order to best attract buyers and so increase their expected profits. In this paper, we consider this issue in terms of setting the seller's reserve price (since the role of the reserve price has received attention in both competitive and non-competitive settings). In particular, we extend the existing analysis by considering how sellers may improve their profit by shill bidding (i.e. bidding within their own auction as a means of setting an implicit reserve price). Moreover, we investigate how the institution can deter this undesirable shill bidding through the use of appropriate auction fees.

\section{MODEL OF COMPETING SELLERS}

The model of competing sellers proceeds in four stages (see figure 1). First, the mediator (an institution such as eBay or Yahoo! that runs the auctions) announces the auction fees to the sellers. The sellers then simultaneously post their reserve prices in the second stage. In the third stage, the buyers simultaneously select an auction (or, equivalently, a seller) based on the observed reserve prices. In the final stage, the buyers (and possibly the sellers who are shill bidding) submit bids and the auctions are executed concurrently.

\section{ANALYSIS}

A complete analysis of equilibrium behaviour and market efficiency for the complete model is intractable [3]. Therefore, in this section, we analyse a simplified version with 
two sellers and no auction fees (in section 4 we address the complete model). We assume that there are $N$ risk neutral buyers, each of whom requires just one item. Each buyer has valuation $v$ independently drawn from a commonly known cumulative distribution $F$ with density $f$ and support $[0,1]$. Each risk neutral seller offers one item for sale, has production costs $x_{i}$, and decides upon a reserve price $r_{i}$ and shill bid $s_{i}$. The preferences of buyers and sellers are described by von Neumann and Morgenstern utility functions.

\subsection{Buyer Equilibrium Behaviour}

The buyers' behaviour for two sellers has been analysed in [2]. A rational buyer with valuation $v<r_{1}$ will not attend any auction. Furthermore, if $r_{1}<v<r 2$, the buyer will always go to seller 1 . The interesting case occurs when $v>r 2$. In a symmetric Nash equilibrium, there is a unique cut-off point $1 \geq w \geq r 2$ where buyers with $v<w$ will always go to seller 1 , and buyers with $v \geq w$ will randomize equally between the two auctions. The cut-off point $w$ is exactly where a buyer's expected utility is equal for both auctions, and is thus found by solving:

$r_{1} \mathcal{F}\left(r_{1}, w\right)^{N-1}+(N-1) \int_{r_{1}}^{w} y \mathcal{F}(y, w)^{N-2} d F(y)=r_{2} \mathcal{F}(w, w)^{N-1}$ where $\mathcal{F}(y, w)=F(y)+[1-F(w)] / 2$. Given the buyers' cutoff point, we can now calculate the sellers' expected revenue.

\subsection{Seller Equilibrium Behaviour}

To calculate the equilibrium behaviour of the sellers, we derive a general expression for the sellers' expected utility. This is calculated by considering the probability of one of three events occurring: (i) no bidders having valuations above the reserve price and the item does not sell, (ii) only one bidder having a valuation above the reserve price and the item sells at the reserve price, or (iii) two or more bidders having valuations above the reserve price and the item sells at a price equal to the second highest valuation. Thus, the expected utility of seller $i$ who has a production cost of $x_{i}$ and sets a reserve price of $r_{i}$ is

$$
\begin{aligned}
& U_{i}\left(r_{i}, x_{i}\right)=N\left(r_{i}-x_{i}\right) \mathcal{G}\left(r_{i}\right)\left(1-\mathcal{G}\left(r_{i}\right)\right)^{N-1} \\
& \quad+N(N-1) \int_{r_{1}}^{1}\left(x_{i}-y\right) \mathcal{G}^{\prime}(y) \mathcal{G}(y)(1-\mathcal{G}(y))^{N-2} d y
\end{aligned}
$$

where $\mathcal{G}(y)$ is the probability that a bidder is present in the auction and that this bidder has a valuation greater than $y$.

Now, in the standard auction with no competing sellers, we have the standard result that $\mathcal{G}(y)=1-F(y)$ and $\mathcal{G}^{\prime}(y)=-f(y)$. However, for two competing sellers, we must account for the fact that the number and valuation of the bidders in the auction is determined by the bidders' cut-off point $w$. Thus, for sellers 1 and 2 (where seller 1 has the lower reserve price), $\mathcal{G}_{1}$ and $\mathcal{G}_{2}$ are given by:

$$
\mathcal{G}_{1}(y)=\left\{\begin{array}{ll}
\frac{1+F(w)}{2}-F(y) & y<w \\
\frac{1-F(y)}{2} & y \geq w
\end{array} \quad \mathcal{G}_{2}(y)= \begin{cases}\frac{1-F(w)}{2} & y<w \\
\frac{1-F(y)}{2} & y \geq w\end{cases}\right.
$$

Thus, the sellers' expected utility depends on the reserve price of both sellers and the equilibrium behaviour is complex. We now apply this result to three different cases: (i) where both sellers declare public reserve prices, (ii) where one seller declares a public reserve price and the other submits a shill bid, and (iii) where both sellers shill bid ${ }^{1}$.

${ }^{1}$ When a seller shill bids, the declared reserve price has no additional benefit. Thus we assume they declare no reserve

\begin{tabular}{|c|c|c|c|}
\hline & $\overline{\mathrm{RP}}$ & $0.452,0.189$ & $0.403,0.220$ \\
\hline & SB & $0.457,0.188$ & $0.423,0$. \\
\hline
\end{tabular}
price (or, equivalently, declare a zero reserve price).
Seller 2

Table 1: Sellers' expected utility when either declaring a reserve price (RP) or to shill bidding (SB).

Both Sellers Announce Public Reserve Prices. In this case, the equilibrium strategy of each seller is given by a Nash equilibrium at which each seller's reserve price is a utility maximising best response to the reserve price of the competing seller. When $x_{1}=x_{2}$, no pure strategy Nash equilibrium exists [2]. However, when the sellers have sufficiently different production costs, we find that a pure Nash equilibrium exists where the reserve price of both sellers is higher than their production costs. We find this equilibrium numerically by iteratively discretising the space of possible reserve prices. That is, for all possible values of $r_{1}$ and $r_{2}$ that satisfy the conditions $x_{1} \leq r_{1} \leq 1$ and $r 1 \leq r_{2} \leq 1$, we calculate $w$ and hence the expected utility of the two sellers. We then search these reserve price combinations to find the values of $r_{1}^{*}$ and $r_{2}^{*}$ that represents the utility maximising best responses to one another. By iterating the process and using a finer discretisation at each stage, we are able to calculate the Nash equilibrium to any degree of precision. The outcomes show that the symmetric case is very much a special case, and the majority of possible production cost combinations yield unique pure strategy Nash equilibria.

One Seller Shill Bids. Rather than announce a public reserve price, either seller may choose to announce a reserve price of zero to attract bidders, and then submit a shill bid to prevent the item from selling at too low a price. Thus, the seller who does not shill bid (seller 2 since $r_{2}$ will be greater than $r_{1}$ ) should declare a reserve price that is a best response to the zero reserve price announced by the bidder who does shill bid. This reserve price is simply given by the value of $r_{2}$ that maximises $U_{2}\left(r_{2}, x_{2}\right)$, given that we calculate $\mathcal{G}_{2}(y)$ as in equation 2 and take $r_{1}=0$ in order to calculate $w$. Given the best response reserve price of seller 2 , and the resulting value of $w$, we can also calculate the shill bid that seller 1 should submit in order to maximise its own expected utility. By substituting $s_{1}$ for $r_{1}$ in equation 1 , and using $\mathcal{G}_{1}(y)$ as given in equation 2 , we find the shill bid that maximises $U_{1}\left(s_{1}, x_{1}\right)$.

Both Sellers Shill Bid. Finally, when both sellers declare a zero reserve price and shill bid, the bidders will randomise equally between either auction, since there is no reserve price information to guide their decision. Thus we find the equilibrium shill bids of both sellers by again substituting $s_{i}$ for $r_{i}$ in equation 1 and hence finding the value of $s_{i}$ that maximises $U_{i}\left(s_{i}, x_{i}\right)$ when $w=0$.

Table 1 shows an example of the resulting four strategy combinations as a normal form game (in this case $N=10$, $x_{1}=0.25$, and $\left.x_{2}=0.5\right)$. Note that both sellers have a dominant strategy to submit shill bids (this result holds in general in the absence of auction fees). At this equilibrium, seller 2 achieves its maximum possible utility. However, seller 1 receives more when neither seller shill bids and is thus better off with a mechanism that deters all parties from submitting shill bids. 


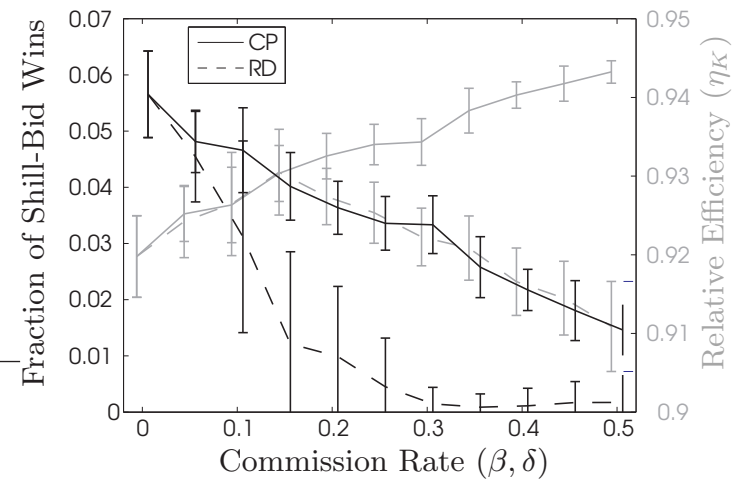

Figure 2: Average fraction of shill-bid wins (black) and relative efficiency (grey) for CP \& RD auction fees $\left(N=10\right.$ and $\left.x_{1}=x_{2}=0\right)$. The error-bars indicate the standard deviation.

\section{AUCTION FEES}

We now consider auction fees and market efficiency in the competing sellers game. We compare two types of auction fees: a closing price $(\mathrm{CP})$ fee that is a fraction, $\beta$, of the selling price (where $\beta$ is the $\mathrm{CP}$ commission rate), and a reserve-difference (RD) fee that is calculated as a fraction, $\delta$, of the difference between the selling price and the seller's declared reserve price, (where $\delta$ is the RD commission rate). The first type of fee is the most common in online auctions such as eBay, Yahoo! and Amazon. The second type of fee was introduced in previous literature, and is shown to prevent shilling for particular bidder valuation distributions in a single multi-stage auction [4].

Auction fees add considerable complexity to the analysis of the competing sellers game since a seller now needs to optimally set both the reserve price and the shill bid. Therefore, we investigate auction fees using a simulation based on evolutionary algorithms (EAs) [1]. The EA maintains a population of possible seller strategies, where a strategy determines the shill bid and reserve price for each auction. At each generation, $M$ seller strategies are randomly selected from the population and compete against one another in a number of consecutive games. The fittest strategies survive and are transferred to the next generation, whereas poor performing strategies are removed from the population. New strategies are explored by slightly modifying existing individuals using a mutation operator. This evolutionary process is repeated for a fixed number of iterations.

We compare auction fees by considering the fraction of shill-bid wins (i.e., the fraction of auctions that result in a shill bid being the highest bid), and a measure of the relative efficiency $\eta_{K}$ of an allocation $K$, where $\eta_{K}$ is given by:

$$
\eta_{K}=\frac{\sum_{i=1}^{N} v_{i}(K)+\sum_{i=1}^{M}\left(x_{i}-x_{i}(K)\right)}{\sum_{i=1}^{N} v_{i}\left(K^{*}\right)+\sum_{i=1}^{M}\left(x_{i}-x_{i}\left(K^{*}\right)\right)},
$$

where $K^{*}=\arg \max _{k \in \mathcal{K}}\left[\sum_{i=1}^{N} v_{i}(k)-\sum_{i=1}^{M} x_{i}(k)\right]$ is an efficient allocation, $\mathcal{K}$ is the set of all possible allocations, $v_{i}(k)$ is bidder $i$ 's utility for an allocation $k \in \mathcal{K}$, and $x_{i}(k)$ is seller $i$ 's production costs for a given allocation (in order to prevent a negative value we add production costs $x_{i}$ in both the denominator and the numerator).

Now, in order to directly compare the auction fees, we first consider the case without production costs. To this end, figure 2 shows the fraction of shill-bid wins and relative efficiency for the two types of auction fees. These results show that the RD fee is better able to reduce shill bidding for this setting. However, in case of high commission rates, a higher efficiency is obtained using the CP fee. Since the RD fee is effectively a combination of a $\mathrm{CP}$ fee and a negative $\mathrm{RD}$ fee, it rewards a high reserve price. If the commission rate is sufficiently high, the reserve price is set higher than the equilibrium price without any auction fees, and thus, results in an inefficient outcome. The $\mathrm{CP}$ fee, on the other hand, is neutral with regard to the reserve price.

In case of production costs we find similar results. However, if both production costs and CP commission rates are very high (e.g. above 30\%), a sharp decline in efficiency is observed using the $\mathrm{CP}$ fee. A seller will then set a very high reserve price to prevent making a loss in case it sells, and, as a result, efficiency becomes very low. Using the RD fee, on the other hand, efficiency does not decrease because sellers never make a loss as long as they report a reserve price above their production costs. The latter type of fee is therefore more robust to high production costs.

To conclude, the experiments show that in most cases highest efficiency is obtained by the commonly applied CP auction fees. The RD auction scheme, however, is more effective in deterring shill bidding and is also more robust to high production costs. This is consistent with earlier results showing that RD auction fees can deter shill bidding for isolated auctions [4]. However, our results show, for the first time, that these fees are also effective for a setting where sellers compete and in case of production costs. Moreover, we see that, when using the $\mathrm{RD}$ fee, sellers pay much less to the mediator overall compared to $\mathrm{CP}$ auction fees. The latter is especially important in a larger setting where multiple mediating institutions compete to attract sellers, since high fees will deter potential sellers.

\section{CONCLUSIONS}

Traditionally, competition among sellers has been ignored when designing auctions and setting auction parameters. However, when faced with competition, we have shown that auction parameters are important in determining the number and type of buyers that are attracted to an auction. We have also shown that such competition provides an incentive for sellers to shill bid, but this can be avoided by a mediator that applies appropriate auction fees. These results are particularly relevant for online markets and multi-agent systems, where competition is strong due to the ease with which a buyer (or a software agent) can search for particular goods. Thus, in these settings, our results can be used by sellers seeking to maximise their profit, or alternatively, by the auction institution itself, who wishes to use appropriate auction fees to deter shill bidding and thus increase the efficiency of the market as a whole.

\section{REFERENCES}

[1] S.M. Bohte, E. Gerding, and J.A. La Poutré. Market-based recommendation: Agents that compete for consumer attention. ACM Transactions on Internet Technology, 4:420-448, 2004.

[2] R. Burguet and J. Sákovics. Imperfect competition in auction design. International Economic Review, 40(1):231-247, 1999.

[3] R. Preston McAfee. Mechanism design by competing sellers. Econometrica, 61(6):1281-1312, 1993.

[4] W. Wang, Z. Hidvégi, and A.B. Whinston. Shill-proof fee (SPF) schedule: The sunscreen against seller self-collusion in online english auctions. Working Paper, 2004. 